Revue de droit comparé du travail et de la sécurité sociale

$3 \mid 2020$

La Directive 2019/1158 du 20 juin 2019 concernant l'équilibre entre vie personnelle et vie privée des parents et des aidants

La situation des parents et des proches aidants en droit suisse à la lumière de la Directive (UE) 2019/1158 du Parlement européen et du Conseil du 20 juin 2019 concernant l'équilibre entre vie professionnelle et vie privée des parents et des aidants

\title{
Stéphanie Perrenoud
}

\section{CpenEdition}

Journals

Édition électronique

URL : https://journals.openedition.org/rdctss/987

DOI : $10.4000 /$ rdctss.987

ISSN : 2262-9815

\section{Éditeur}

Centre de droit comparé du travail et de la sécurité sociale

Édition imprimée

Date de publication : 1 novembre 2020

Pagination : 132-143

ISSN : $2117-4350$

Référence électronique

Stéphanie Perrenoud, "La situation des parents et des proches aidants en droit suisse à la lumière de la Directive (UE) 2019/1158 du Parlement européen et du Conseil du 20 juin 2019 concernant

l'équilibre entre vie professionnelle et vie privée des parents et des aidants », Revue de droit comparé du travail et de la sécurité sociale [En ligne], 3 | 2020, mis en ligne le 01 novembre 2021, consulté le 11 novembre 2021. URL : http://journals.openedition.org/rdctss/987 ; DOI : https://doi.org/10.4000/ rdctss. 987

\section{(c) (†) $\ominus$}

Revue de droit comparé du travail et de la sécurité sociale est mise à disposition selon les termes de la Licence Creative Commons Attribution - Pas d'Utilisation Commerciale - Pas de Modification 4.0 International. 


\section{LA DIRECTIVE 2019/1158 CONCERNANT L'ÉQUILIBRE ENTRE VIE PROFESSIONNELLE ET VIE PRIVÉE DES PARENTS ET DES AIDANTS AU ROYAUME-UNI : L'EFFET BREXIT}

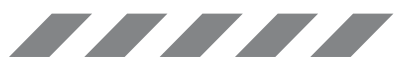

\section{ABSTRACT}

The directive on Work Life Balance will be one of the EU social policy measures not applicable in the UK in the post Brexit environment. In this field, UK workers already benefit from a number of rights found in the new EU instrument however, or even more generous entitlements. Some of the EU directive provisions would nevertheless constitute new and welcome developments the UK workplace.

KEYWORDS: Work/Life Balance, Brexit, Paternity Leave, Parental Leave, Flexible Working, Carers' Leave, Shared Parental Leave.

\section{RÉSUMÉ}

La directive concernant l'équilibre entre vie professionnelle et vie privée sera l'une des premières mesures de droit social européen à ne pas s'appliquer au Royaume-Uni dans l'environnement postBrexit. Dans ce domaine, les employés britanniques bénéficient cependant déjà d'un certain nombre de droits issus de la nouvelle directive, ou même de protection plus généreuse. Certaines des provisions de la nouvelle directive constitueraient néanmoins de nouveaux développements bienvenus.

MOTS CLÉS: Équilibre entre vie professionnelle et vie privée, Brexit, congé de paternité, congé parental, travail flexible, congé d'aidant, congé parental partagé. 
a sortie du Royaume-Uni de I'UE le 31 janvier 2020, à la suite du référendum de juin 2016, a remis en question la politique et la législation en matière de conciliation de la vie professionnelle et familiale au Royaume-Uni1. Sauf accord contraire, le Royaume-Uni ne sera pas tenu de mettre en œuvre la directive 2019/1158 sur l'équilibre entre la vie professionnelle et la vie privée des parents et des aidants ${ }^{2}$ dans la mesure où la date de transposition du 2 août $2022^{3}$ (ou le 2 août 2024 s'agissant des dispositions relatives à la rémunération parentale ${ }^{4}$ ) se situe au-delà de la période transitoire - qui se termine le 31 décembre 2020 - au cours de laquelle le Royaume-Uni reste soumis au droit de $I^{\prime} U E^{5}$. Le présent article analyse dans quelle mesure la législation actuelle pourrait déjà satisfaire, voire dépasser, les exigences de la directive 2019/1158, et étudie de quelle façon les parents travaillant au Royaume-Uni pourraient gagner à bénéficier d'un alignement de la législation britannique sur cette directive européenne.

\section{I - LE CONGÉ DE PATERNITÉ}

Récemment encore, le Royaume-Uni offrait aux pères et aux partenaires qui travaillent plus de droits que ne l'exigeait la législation de I'UE, laquelle encourageait simplement les Etats membres à leur reconnaître un droit distinct à bénéficier d'un congé de paternité ou d'adoption ${ }^{6}$. Le droit légal à un congé de paternité rémunéré a été introduit au Royaume-Uni par la loi sur l'emploi de 2002, qui a pris effet en avril 2003. A certains égards, les dispositions de la législation britannique sur le congé de paternité sont similaires aux exigences de la directive 2019/1158, qui prévoit 10 jours de congé de paternité à l'occasion de la naissance d'un enfant, à prendre avant ou après la naissance ${ }^{7}$.

Au Royaume-Uni, les pères qui travaillent ont droit à une ou deux semaines consécutives de congé de paternité, qui peuvent être prises dans les 56 jours suivant la naissance de l'enfant $^{8}$. La rigidité relative du dispositif de congé de paternité au Royaume-Uni, ainsi

1 Les dispositions existantes du droit du travail dérivées de l'UE ont été conservées, mais pourraient être modifiées à l'avenir, conformément à la procédure législative britannique (EU Withdrawal Act 2018, Art. 2(1) et Art. 7). Voir également J. Kenner, « Brexit and Labour Standards at the time of COVID-19 - To Converge or to Diverge, that is the Question », Eulawanalysis, 19 juin 2020 : http://eulawanalysis.blogspot.com/2020/06/brexit-and-labour-standards-at-time-of.html

2 Directive (UE) 2019/1158 du Parlement européen et du Conseil du 20 juin 2019 relative à l'équilibre entre la vie professionnelle et la vie privée des parents et des aidants et abrogeant la directive 2010/18 / UE du Conseil [2019] JO L188 / 79.

3 Art. 20, § 1, de la directive 2019/1158.

4 Art. 20, § 2, de la directive 2019/1158.

5 Art. 127 de l'accord de retrait entre I'UE et le Royaume-Uni [2020] JO L29/7.

6 Résolution du Conseil du 29 juin 2000 sur la participation équilibrée des femmes et des hommes à la vie familiale et professionnelle. JO [2000] C218; Art. 7 de la directive 76/207/CEE du Conseil du 9 février 1976 relative à la mise en œuvre du principe de l'égalité de traitement entre hommes et femmes en ce qui concerne l'accès à l'emploi, à la formation et à la promotion professionnelles et les conditions de travail, telle que modifiée par la directive 2002/73/CE du Parlement européen et du Conseil (JO L 269/15).

7 Art. 4 de la directive 2019/1158.

8 The Paternity and Adoption Leave Regulations 2002 (SI $2002 \mathrm{n}^{\circ} 2788$ ), reg. 5. 
que la faible rémunération accordée pendant ce congé, ne sont pas en contradiction avec la directive puisque cette dernière laisse aux Etats membres la liberté de déterminer la souplesse du recours à ce droit ${ }^{9}$ et lie le taux de rémunération à celui des indemnités de maladie ${ }^{10}$. L'indemnité accordée peut même dépasser les exigences de la directive 2019/1158. Les pères et partenaires britanniques ont droit à une indemnité de paternité au taux légal (actuellement 151,20f) ou 90\% du salaire hebdomadaire moyen, si ce dernier montant est moins élevé. L'indemnité pour congé de paternité est plus élevée que l'indemnité de maladie (actuellement 95,85f), ce qui est conforme aux exigences de la directive.

Dans le même temps, le paragraphe 30 des considérants de la directive encourage les Etats membres à prévoir une rémunération ou une allocation pour le congé de paternité, qui soit égale à celle prévue pour le congé de maternité. Une telle mesure serait avantageuse pour les pères dans la mesure où les 6 premières semaines de l'indemnité légale de maternité au Royaume-Uni sont payées à $90 \%$ du salaire hebdomadaire moyen. Mais il semble fortement improbable que le gouvernement britannique actuel s'inspire de la directive en alignant les rémunérations de maternité et de paternité. En effet, une proposition de la Commission parlementaire pour les femmes et les égalités, selon laquelle les 4 premières semaines de congé de paternité devraient être rémunérées à $90 \%$ de la rémunération hebdomadaire - avec un plafond pour les hauts revenus - a été rejetée ${ }^{11}$.

Néanmoins, en raison des pressions exercées par la Commission pour l'égalité et les droits de l'homme ${ }^{12}$ et le groupe de défense des familles «Working Families »" en faveur de l'introduction d'un congé de paternité autonome et bien rémunéré, le gouvernement est revenu en 2019 sur l'examen du congé de paternité et de la rémunération dans sa consultation sur les mesures visant à soutenir les familles ${ }^{14}$, consultation qui citait spécifiquement la directive européenne comme point de comparaison. Par conséquent, la possibilité d'un alignement sur la directive et d'une amélioration des conditions de rémunération du congé de paternité ne peut être exclue.

Une analyse historique du congé de paternité au Royaume-Uni révèle que son degré de flexibilité et son niveau de rémunération sont des facteurs importants pour favoriser un recours utile et efficace à de tels dispositifs. La rigidité du calendrier et des modalités du congé de paternité, en sus du faible taux de rémunération, expliqueraient le faible nombre de pères qui en bénéficient. Le lien entre le nombre de pères prenant un congé de paternité

9 Art. 4, § 1, de la directive 2019/1158.

10 Art. 8, § 2, de la directive 2019/1158.

11 Commission pour les femmes et les égalités, Chambre des communes, premier rapport de session 2017-2019, Fathers and the workplace, WESC, 2018, p. 16: https://publications.parliament.uk/pa/ $\mathrm{cm} 201719 / \mathrm{cmselect} / \mathrm{cmwomeq} / 358 / 358$.pdf

12 EHRC, Women's rights and gender equality in 2018 : update report, Equality and Human Rights Commission, 2018 : www.equalityhumanrights.com/en/womens-rights-and-gender-equality-2018update-report

13 Working Families, "Increase the Joy: Improving Shared Parental Leave », 2018 : www. workingfamilies.org.uk/wp-content/uploads/2018/05/WF-Briefing-2018-Increase-the-Joy-waysto-improve-Shared-Parental-Leave-FINAL.pdf

14 "Good Work Plan : Proposals to support families », juillet 2019 : https://beisgovuk.citizenspace. $\mathrm{com} / \mathrm{m} / 9 \mathrm{e} 55319 \mathrm{a} / \mathrm{supporting} \mathrm{documents/goodworkfamilysupportconsultation.pdf}$ 
et la rémunération accordée a ainsi été clairement établi ${ }^{15}$. Une enquête commandée par I'EHRC a révélé que $55 \%$ des nouveaux pères ont pris un congé de paternité pour passer du temps avec leur nouveau-né et leur partenaire. Sur les $45 \%$ qui n'ont pas pu le faire, $66 \%$ ont déclaré qu'ils l'auraient souhaité, le motif d'empêchement de prendre un congé étant le plus souvent d'ordre financier ${ }^{16}$. Plus le congé est long, plus la rémunération est un facteur de décision ${ }^{17}$. Cela signifie qu'en termes de rémunération du congé de paternité, ni les exigences de la directive européenne, ni la législation britannique, ne permettent aux pères de devenir des partenaires assumant un rôle égal dans l'éducation des enfants.

L'un des points sur lesquels les pères britanniques gagneraient à bénéficier de la nouvelle directive réside dans le fait que le congé de paternité, en vertu de la législation de I'UE, peut être pris dès le premier jour ${ }^{18}$, bien que la directive permette aux Etats membres de subordonner le droit à une rémunération ou allocation à des périodes d'emploi antérieures ne dépassant pas 6 mois ${ }^{19}$.

Dans la législation britannique, le droit au congé de paternité et le droit à une rémunération sont subordonnés au fait que le père soit employé sans interruption depuis au moins 26 semaines avant la $14^{\text {e }}$ semaine précédant la semaine prévue de l'accouchement. Cette condition d'ancienneté a privé 44000 pères de ces droits en $2016^{20}$.

En outre, la directive pourrait permettre à davantage de pères britanniques de bénéficier d'un congé de paternité puisqu'elle s'applique à tous les travailleurs qui ont un contrat de travail ou une relation de travail au sens de la législation, des conventions collectives ou de la pratique en vigueur dans chaque Etat membre, en tenant compte de la jurisprudence de la Cour de justice ${ }^{21}$. Au Royaume-Uni, seuls ceux qui ont un statut officiel de salarié ont droit à un congé de paternité et à une rémunération, ce qui exclut généralement les travailleurs occasionnels de la gig economy ${ }^{22}$.

Une interprétation large du "statut de salarié » n'excluant que les travailleurs indépendants, déjà adoptée par la Cour dans le cadre du travail à temps partiel ${ }^{23}$, permettrait de remettre en question la portée plus réduite du congé de paternité dans la législation britannique.

15 J. Lewis et M. Campbell, « UK Work/Family Balance Policies and gender Equality 1997-2005 », Social Policies: International Studies in Gender, State and Society, 2007, vol. 4, p. 14 ; G. Games, The Legal Regulation of Pregnancy and Parenting in the Labour Market, Routledge, 2009, p. 43.

16 Equality and Human Rights Commission, Working Better: Fathers, family and work - contemporary perspective : http://www.equalityhumanrights.com/uploaded files/research/41 wb fathers family and work. pdf

17 Promoting Uptake of Parental leave and Paternity Leave Among Fathers in the European Union, Eurofoundation, Office des publications de l'Union Européenne, 2015.

18 Art. 4, § 2, de la directive 2019/1158.

19 Art. 8, § 2, de la directive 2019/1158.

20 Fathers and the Workplace, op. cit., p. 16.

21 Art. 2 de la directive 2019/1158.

22 Fathers and the Workplace, op. cit., p. 16.

23 Affaire C-393/10 O'Brien v Ministry of Justice ECLI: EU:C:2012:110, § 44. Voir également N. Kountouris, "The Concept of 'Worker' in European Labour Law: fragmentation, autonomy and scope », Industrial Law Journal, 2018, vol. 47/2, p. 192. 


\section{II - LE CONGÉ PARENTAL}

En vertu de la directive européenne, chaque parent a droit individuellement à 4 mois de congé parental pour chaque enfant jusqu'à l'âge de ses 8 ans, dont 2 mois ne peuvent être transférés d'un parent à l'autre. La loi britannique prévoit déjà des dispositions plus généreuses : les salariés ayant la responsabilité d'un enfant ont ainsi droit à 18 semaines de congé pour chaque enfant, et ce jusqu'au $18^{\mathrm{e}}$ anniversaire de l'enfant ${ }^{24}$. La durée plus longue du congé et de l'âge de l'enfant prévues par la législation britannique garantissent que les deux parents sont en mesure d'exercer leur droit de manière effective et sur un pied d'égalité, comme l'exige la directive ${ }^{25}$. L'obligation liée à une année de travail continu est également conforme à la période de qualification maximale prévue par la directive ${ }^{26}$.

Selon la réglementation britannique, le congé parental n'est pas transférable et reflète donc l'incitation " à l'utiliser sous peine de le perdre ", ce qui sous-tend la nontransférabilité d'au moins deux mois imposée par la directive ${ }^{27}$. La rigidité de la législation britannique a été critiquée puisqu'elle impose de prendre le congé parental par tranches minimales d'une semaine ${ }^{28}$, mais elle reste cependant conforme à la directive. Si l'article 5, paragraphe 6, établit que les parents doivent avoir le droit d'exercer leurs droits de façon flexible, les Etats membres peuvent préciser les modalités de mise en œuvre de ce droit.

L'avantage de la mise en œuvre de la directive au Royaume-Uni, ou d'un alignement sur des dispositions, consisterait en l'introduction du droit à une indemnité parentale ${ }^{29}$ puisque, actuellement, le congé parental n'est pas rémunéré au Royaume-Uni. Le gouvernement s'inquiète du coût pour le contribuable dans la mesure où les employeurs britanniques peuvent se faire rembourser au moins $92 \%$ de la rémunération légale versée dans le cadre des mesures en faveur de la famille, par le biais du système fiscal et des cotisations sociales. Dans le cas des petites entreprises, $103 \%$ du coût de l'allocation réglementaire peut être reversé par l'état. Le coût pour les entreprises est également pris en compte, car l'absence du travail représente un coût pour l'employeur, à la fois en termes de couverture du travail du salarié en congé (y compris le recrutement de travailleurs intérimaires) et de formation d'autres membres du personnel pour faire son travail ${ }^{30}$.

Par conséquent, l'objectif principal de la révision actuelle du congé parental est de trouver un équilibre entre la durée du congé, le droit à une indemnité et le montant de celle$c i$, et la perturbation de l'activité des entreprises ${ }^{31}$. Le résultat involontaire d'un alignement sur la directive pourrait être l'introduction du taux de rémunération minimum accompagné d'un raccourcissement de la durée du congé ou d'une réduction de l'âge limite des enfants, ce qui, dans l'ensemble, pourrait ne pas favoriser le recours au congé parental.

24 The Maternity and Parental Leave Regulations, 1999. SI 1999 n³312, regs.13-15.

25 Art. 5, § 1, de la directive 2019/1158.

26 Art. 5, § 4, de la directive 2019/1158.

27 Art. 5, § 2, de la directive 2019/1158.

28 South Central Trains v Rodway [2005] ICR 1162.

29 Art. 8, § 3, de la directive 2019/1158.

30 «Good Work Plan: Proposals to supportfamilies' consultation », Juillet 2019, p. 28 : https://beisgovuk. citizenspace.com/lm/9e55319a/supporting documents/goodworkfamilysupportconsultation.pdf

31 Ibid., p. 27. 
Toute comparaison entre la directive et la réglementation britannique actuelle sur les droits des pères qui travaillent est encore compliquée par l'existence d'un droit hybride au congé parental partagé (SPL) lequel, bien que basé sur le « congé de paternité supplémentaire $(A P L){ }^{32}$, ne constitue pas un droit individuel du père. Le congé parental partagé ayant été introduit en plus des dispositions sur le congé de paternité et le congé parental non rémunéré, la possibilité d'une incompatibilité avec la nouvelle directive semble limitée ${ }^{33}$. Selon la jurisprudence de la CJUE, lorsque les Etats membres choisissent d'introduire des droits supplémentaires, plus favorables que les exigences minimales de la directive de l'UE, rien ne les empêche de fixer des conditions d'exercice de ces droits plus généreux ${ }^{34}$.

Le congé parental partagé a été introduit par la loi de 2014 sur les enfants et les familles, à la suite de la Consultation sur les entreprises modernes lancée en mai 2011 par le gouvernement de coalition en vue d'une réforme globale des congés de maternité, de paternité et parental, afin d'introduire une gamme de mesures plus flexibles qui permettrait aux parents de partager les congés et les indemnités de manière adaptée à leur situation personnelle. Les parents ont désormais droit à un congé parental partagé d'une durée maximale de 50 semaines, qui peut être partagé de manière flexible par les pères et les mères qui travaillent, en plus du droit individuel des pères à 2 semaines de congé de paternité. II peut être pris par les parents en même temps ou à tour de rôle, avant le premier anniversaire de l'enfant, et est rémunéré jusqu'à 37 semaines, au même taux que le congé de paternité 35 .

Ce congé répondait à la critique de la rigidité des conditions requises pour la prise d'un congé supplémentaire de paternité ( $A P L)$, qui permettait aux mères et aux pères un choix formel dans les rôles parentaux, mais sans réelle flexibilité : le père ne pouvait prendre l'APL que si la mère retournait au travail.

En outre, le droit du père à l'APL était subordonné à la condition que la mère ait droit légalement à un congé ou à une allocation de maternité. Le calendrier était également contraignant : les 6 premiers mois après la naissance étaient réservés à la mère et ne devaient pas être partagés, mais le congé de paternité supplémentaire devait être pris avant le premier anniversaire de l'enfant. En raison de son caractère subordonné et de ces conditions peu favorables, l'APL n'a pas vraiment réussi à apporter plus de flexibilité aux dispositions en faveur de la famille dans le droit du travail et a renforcé l'idée que les pères sont des « partenaires secondaires $»^{36}$.

Malheureusement, si le remplacement du congé de paternité supplémentaire par un congé parental partagé a amélioré la flexibilité, des enquêtes récentes montrent que le

32 Le droit de prendre 26 semaines supplémentaires de congé de paternité a été introduit en 2011 par le Work and Families Act 2006, remplacé par le Shared Parental Leave en 2015.

33 Voir J. Atkinson, "Shared Parental Leave in the UK: can it advance gender equality by changing fathers into co-parents? ", International Journal of Law in Context, 13(3), 2017, p. 356.

34 Affaire 337/10 Neidel v Stadt Frankfurt am Main ECLI:EU:C:2012:263.

35 Shared Parental Leave Regulations 2014. SI n³050; The Statutory Shared Parental Pay (General) Regulations 2014, SI n³051.

36 Voir E. Caracciolo di Torella, « New Labour, New Dads - The Impact of Family - Friendly Legislation on Fathers", Industrial Law Journal, 2007, 36/3, p. 318 ; G James, "The Work and Families Act 2006: Legislation to Improve Choice and Flexibility », Industrial Law Journal, 2006, 35(3), p. 272. 
recours au congé parental partagé n'a pas dépassé les $1 \%$ ou $2 \%{ }^{37}$. Cela peut être attribué à plusieurs lacunes dans la conception de ce droit, qui impactent principalement les pères.

Premièrement, le droit au congé parental partagé dépend de la décision de la mère de réduire son congé de maternité et son allocation de maternité. Cela perpétue le phénomène de "contrôle maternel " issu des normes de genre dans les constructions culturelles liées aux obligations familiales ${ }^{38}$, sapant ainsi le concept de l'égalité du père en tant que partenaire dans l'éducation des enfants. De plus, les pères célibataires n'ont pas droit au congé parental partagé.

En second lieu, la complexité des modalités d'éligibilité au congé parental partagé réduit son utilité pour les pères. Pour avoir le droit de demander un congé partagé, la mère ou le père doivent être salariés et satisfaire au critère de continuité de l'emploi (au moins 26 semaines avant la fin de la $15^{\mathrm{e}}$ semaine précédant la date prévue pour la naissance (ou la date d'accueil d'un enfant adopté). Si la mère remplit les conditions d'admissibilité, le père doit encore, pour pouvoir partager le congé, réunir les critères de l'emploi ou du travail indépendant, et du seuil de rémunération hebdomadaire.

En troisième lieu, les conditions d'éligibilité à une indemnité de congé parental partagé sont complexes et décourageantes. Pour avoir droit à l'indemnité parentale partagée, les pères doivent avoir droit à l'indemnité de paternité. En outre, le droit du père à une allocation dépend du droit de la mère à une allocation ou indemnité de maternité ou d'adoption. Si le droit au congé parental partagé était accordé dès le premier jour pour les pères, conformément à la directive 2019/1158, il serait sans nul doute d'un grand pas en avant.

Par ailleurs, la faible rémunération du congé parental partagé creuse le fossé social entre les travailleurs ayant un niveau élevé de revenus et d'éducation, qui peuvent s'autoriser à prendre un congé parental, et ceux dont les emplois faiblement rémunérés ne leur permettent pas de se passer d'un salaire ${ }^{39}$. Cette inégalité sociale est encore renforcée par les politiques appliquées par les employeurs. Ils peuvent compléter l'indemnité légale par des dispositions contractuelles plus généreuses, mais ils les réservent généralement aux salariés les mieux rémunérés, dans un souci d'attirer et de retenir une main-d'œuvre très qualifié $e^{40}$.

Dans les affaires Ali contre Capita et Hextall, l'argument avancé était que la faible rémunération du congé parental partagé affecte principalement les pères, car les mères peuvent choisir d'exercer leur droit à une indemnité de maternité améliorée pendant

37 H. Birkett et S. Forbes, "Shared Parental Leave: why is take-up so low and what can be done? », University of Birmingham Press Release, août 2019 : https://www.birmingham.ac.uk/news/ latest/2019/08/university-of-birmingham-research-shows-take-up-of-shared-parental-leave-isincreasing.aspx

$38 \mathrm{H}$. Birkett et S. Forbes, « Where is Dad? Exploring the low take-up of inclusive parenting policies in the UK», Policy Studies, 2019, 40/2, p. 205.

39 O. Golynker, "Family-friendly reform of employment law in the UK: an overstretched flexibility », Journal of Social Welfare Law and Family Law, 2015, 37(3), p. 378 ; G James, " Family-friendly Employment Laws (Re)assessed : the potential of care ethics ", Industrial Law Journal, 2016, 45/4, p. 477 ; A. Koslowski et G. Kadar-Satat, « Fathers at Work: explaining the gaps between entitlement to leave policies and uptake », Community, Work and Family, 2019, 22/2, p. 129.

40 H. Birkett et S. Forbes, op. cit., vol. 27, n³8. 
les 6 premières semaines du congé de maternité, avant de passer au congé parental partagé. Cependant, la Cour d'appel a rejeté les arguments de discrimination directe et de discrimination indirecte, estimant qu'un homme ne peutêtre comparéà une femme en congé de maternité car, en vertu de la loi de 2010 sur l'égalité, un congé de maternité est accordé aux nouvelles mères pour les aider à se remettre physiquement et psychologiquement de la grossesse et de l'accouchement. On ne peut établir de comparaison qu'avec une salariée en congé parental partagé (il n'y a pas de différence dans la rémunération) ${ }^{41}$.

A la suite du refus d'un recours par la Cour suprême, la décision de la Cour d'appel sur cette question reste définitive. Cependant, le jugement dans les affaires Ali et Hextall est des plus révélateurs. II démontre en effet que, quel que soit le résultat obtenu dans une telle affaire invoquant la discrimination, il ne sera pas satisfaisant. Une victoire des pères signifierait un nivellement où les mères perdraient leur droit à une indemnité de maternité améliorée ${ }^{42}$. Dans le même temps, cela remettrait en question la justification de la politique d'octroi d'un long congé de maternité, combiné à une augmentation de l'indemnité de maternité en raison de différences physiologiques et autres, entre les mères et les pères ${ }^{43}$. La manière dont le congé parental partagé flexible a été introduit au Royaume-Uni ne permet pas de choix réel, car elle n'a pas tenu compte des contraintes entourant ce choix ${ }^{44}$.

La comparaison avec la directive 2019/1158 donne matière à réflexion. Si l'on devait aligner le congé parental partagé sur les règles du congé parental, l'exigence de continuité d'emploi pourrait être maintenue et le faible taux de rémunération serait conforme à la directive. Néanmoins, il faudrait que deux mois de congé deviennent non transférables entre les parents (et non plus 2 semaines, comme le prévoit la législation britannique actuelle). Mais surtout, l'alignement sur la directive nécessiterait de rendre l'exercice du droit au congé parental partagé par le père indépendant de la situation de la mère.

Le moyen le plus prometteur d'améliorer les droits des pères au Royaume-Uni serait de repenser radicalement le régime des congés de maternité et de paternité, du congé parental et du congé parental partagé, et de les remplacer par un congé parental qui inclurait une période non transférable, des périodes réservées aux mères et aux pères, ainsi que des périodes partagées de manière flexible, avec une rémunération correspondante, à un taux au moins équivalent au taux majoré actuel du congé de maternité légal $(90 \%$ du salaire hebdomadaire) pendant les 6 premières semaines, et une rémunération adéquate pendant les périodes restantes. Dans le même temps, la durée maximale actuelle du congé parental et la limite d'âge des enfants devraient être conservées, bien que la période rémunérée puisse être limitée ${ }^{45}$.

41 Affaires Aliv Capita Customer Management Ltd et Hextall v Chief Constable of Leicestershire Police [2019] EWCA Civ 900, 24 Mai 2019.

42 La malheureuse conséquence de la décision antérieure d'un tribunal écossais de première instance dans l'affaire Snell v Network Rail (ETS/1400178/2016).

43 Ali et Hextall [66]-[67], op. cit.

44 J. Lewis, Work-Family Balance, Gender and Policy, Edward Elgar, 2009, p. 19.

45 Une proposition de révision du régime de tous les droits d'équilibre entre vie professionnelle et vie privée des parents a été présentée dans la consultation « Good Work Plan: Proposals to support families $», n^{\circ} 30$. 


\section{III - LES FORMULES SOUPLES DE TRAVAIL}

Les formules souples de travail font depuis longtemps partie des mesures dont bénéficient les salariés britanniques. Leur portée personnelle et matérielle est également plus large et offre aux travailleurs britanniques un dispositif plus généreux que dans la directive européenne.

Cependant, il existe des différences en ce qui concerne le retour au mode initial de travail à la fin de la période temporaire. II est toutefois prévu que cette question soit revue dans un nouveau projet de loi sur l'emploi. En dépit de son apparente " générosité » plus grande, la législation britannique a également fait l'objet de critiques en raison du grand nombre de motifs sur lesquels les employeurs peuvent s'appuyer pour refuser une demande, ainsi que du manque d'adhésion des pères, malgré la neutralité de genre de ce droit.

Les "formules souples de travail " s'entendent au sens le plus large, comme dans la directive européenne ${ }^{46}$. La législation les définit comme une modification du nombre d'heures travaillées, des horaires de travail imposés ou du lieu de travail ${ }^{47}$. Dans la pratique, cela couvre le travail à temps partiel, le télétravail, le travail en période scolaire, des journées de travail plus longues sur un nombre de jours réduit, des journées débutant et s'achevant plus tôt, etc. ${ }^{48}$.

Le droit de demander des formules de travail souples a été introduit en 2002 par le gouvernement travailliste dans le cadre de sa politique visant à améliorer l'équilibre entre vie professionnelle et vie privée ${ }^{49}$. Dans sa première version, ce droit visait uniquement à permettre aux parents de s'occuper d'un enfant ${ }^{50}$, et n'était donc accessible qu'aux parents d'enfants âgés de 5 ans ou moins. Ce droit était conditionnel - il l'est encore aujourd'hui puisque le salarié concerné devait avoir travaillé pendant 26 semaines avant d'avoir le droit d'en faire la demande ${ }^{51}$.

Si seuls les parents avaient, jusqu'alors, la possibilité de modifier leurs conditions de travail pour faciliter la garde des enfants, il est devenu nécessaire, en raison des évolutions démographiques et du vieillissement de la population, d'accorder le même droit aux salariés s'occupant d'adultes ${ }^{52}$, définis comme des conjoints, des parents et des adultes résidant au sein du même foyer que le salarié ${ }^{33}$.

46 Art. 3, § 1, de la directive 2019/1158 : recours au travail à distance, à des horaires de travail souples ou à une réduction du temps de travail, et voir également la Préambule (34).

47 ERA 1996, s 80F(1) (a).

48 Sur le travail flexible, voir par exemple le site du gouvernement : https://www.gov.uk/flexibleworking/types-of-flexible-working

49 Voir Department of Trade Industry, «Work and Parents: Competitiveness and Choice », Mai 2001. Pour un commentaire sur cette mesure, voir par exemple J. Lewis et M. Campbell, « UK Work/ Family Balance Policies and Gender Equality 1997-2005 », Social Politics: International Studies in Gender, 2007, vol. 14 (1).

50 Employment Act 2002, notes explicatives : http://www.legislation.gov.uk/ukpga/2002/22/notes/ division/ 3/4/1/6

51 ERA 1996, s 80F.

52 Work and Families Act 2006

53 Voir G. James, "The Work and Families Act 2006 : Legislation to Improve Choice and Flexibility », Industrial Law Journal, 2006, vol. 35(3), p. 272. 
Modifiée en 2009, la loi a ensuite étendu le même droit aux parents dont les enfants avaient moins de 17 ans $^{54}$. Sur ce point, les universitaires avaient critiqué la distinction injustifiée faite entre les parents d'enfants plus jeunes ou plus âgés en ce qui concerne l'équilibre entre la famille et le travail ${ }^{55}$ : lorsque les enfants ont plus de 6 ans, les responsabilités familiales seraient tout aussi exigeantes et difficiles à concilier avec les schémas de travail traditionnels. Enfin, en 2014, ce droit a été étendu à tous les salariés, sans qu'il soit nécessaire d'être un aidant, compte tenu du fait que chacun a droit à un équilibre entre vie professionnelle et vie privée, qu'il ait ou non des responsabilités familiales ${ }^{56}$.

A première vue, le fait que tout le monde bénéficie des mêmes droits semblait éliminer les tensions susceptibles d'exister dans l'entreprise entre les aidants et les non-aidants. Cependant, on pourrait souligner qu'à défaut de se concentrer sur ceux qui peuvent subir des préjudices en termes d'avancement professionnel, on crée une difficulté puisque le fait d'être un aidant n'est pas nécessairement un choix et cela désavantage les parents ${ }^{57}$.

Aujourd'hui, tous les salariés peuvent demander une modification de leurs horaires ou de leurs modalités de travail, en vertu de l'article 80F de la loi de 1996 sur les droits en matière d'emploi. L'exigence des 26 semaines d'ancienneté pour accéder à ce droit est restée en vigueur et elle est conforme à la directive européenne actuelle ${ }^{58}$. II s'agit bien d'un droit, mais du droit d'en faire la demande seulement. La procédure de demande et les éventuelles justifications ouvertes aux employeurs pour refuser le changement de régime de travail étaient autrefois réglementées par la loi, mais à ce jour, l'employeur est tenu de traiter la demande " de manière raisonnable ${ }^{59}$, et de donner une réponse au salarié dans les trois mois. Le salarié ne peut faire plus d'une demande par an. L'employeur peut invoquer un certain nombre de motifs pour refuser la demande, qui concernent principalement les coûts et les exigences opérationnelles ${ }^{60}$. II est arrivé que les tribunaux remettent en question les motifs invoqués par les employeurs lorsque les modalités de travail flexibles n'ont pas été acceptées, pour des raisons de coût entre autres ${ }^{61}$.

Pour aider les entreprises et les travailleurs, la législation est complétée par un Code de bonnes pratiques non contraignant ${ }^{62}$, qui explique plus en détails la manière dont les employeurs doivent traiter une demande et qui énumère les bonnes pratiques, telle la mise

54 The Flexible Working (Eligibility, Complaints and Remedies) (Amendment) Regulations 2009, SI 2009/595.

55 Voir par exemple G. James, op. cit., 2006, p. 278.

56 Children and Families Act 2014.

57 Voir par exemple, O. Golynker, "Family-friendly reform of employment law in the UK : an overstretched flexibility », Journal of Social Welfare Law and Family Law, 2015, vol. 37(3), p. 378.

58 Art. 9, § 4, de la directive 2019/1158.

59 ERA 1996, s 80G.

60 La liste complète est énoncée dans la section $80 \mathrm{G}$ (b) : le poids des coûts supplémentaires; effet néfaste sur la capacité à répondre à la demande des clients; incapacité à réorganiser le travail du personnel existant ; incapacité à recruter du personnel supplémentaire ; impact négatif sur la qualité ; impact négatif sur la performance ; insuffisance de travail pendant les périodes où le salarié se propose de travailler; changements structurels prévus.

61 Affaire BA v Starmer [2005] IRLR 862 (EAT), dans laquelle British Airways a refusé la demande d'une femme pilote de travailler à $50 \%$ d'un contrat à temps plein en raison de responsabilités familiales. Cela a été considéré comme une discrimination sexuelle indirecte.

62 ACAS, Code of Practice on handling in a reasonable manner requests to work flexibly (2014). 
en place d'un mécanisme de recours interne pour les salariés si leur demande est rejetée. La procédure prévue dans la législation britannique serait donc conforme aux exigences de la directive consistant à répondre à une demande dans un délai raisonnable et à permettre aux employeurs de justifier un refus ${ }^{63}$.

Un recours n'est disponible que si la procédure n'a pas été suivie ou si le refus est fondé sur des faits erronés. Dans ce cas, le salarié peut intenter une action devant le tribunal du travail dans un délai de trois mois, mais les réparations sont exclusivement financières et plafonnées à huit semaines de salaire. Le salarié est également protégé contre tout préjudice subi pour des raisons liées à la demande de flexibilité du travail ${ }^{64}$ et contre le licenciement ${ }^{65}$, conformément à la directive ${ }^{66}$.

Par rapport à la directive européenne, le droit britannique ne prévoit pas de caractère temporaire des formules souples de travail, en ce sens qu'il ne limite pas la durée des modalités flexibles ${ }^{67}$ et n'accorde pas aux salariés le droit de revenir à leur mode de travail initial ${ }^{68}$ (comme par exemple, repasser du temps partiel au temps plein). Toute modification des horaires de travail est donc une modification permanente du contrat, ce que Golynker appelle le "caractère irréversible de la modification du contrat "69. Le manque d'appui juridique permettant de retrouver un poste à temps plein peut notamment enfermer indéfiniment les femmes dans un poste à temps partiel et, comme le reconnaît la directive, constituer une sanction sur le long terme pour des femmes qui auraient moins cotisé aux régimes de sécurité sociale et de retraite ${ }^{70}$.

Dans un nouveau projet de loi sur l'emploi, le gouvernement actuel envisage de faire des formules souples de travail la position par défaut pour tous les salariés ${ }^{71}$. II ne s'agit encore que d'un projet ${ }^{72}$ et il n'est pas encore très étoffé, mais on peut s'attendre à ce que les mécanismes mis en place en raison de la Covid-19, encourageant le recours au télétravail chaque fois que c'était possible, auront donné bien plus de poids à ce projet de $1 \mathrm{oi}^{73}$.

Les chiffres les plus récents montrent que l'adoption des formules souples de travail a augmenté, mais de façon ralentie au cours de ces dernières années (la proportion de

63 Art. 9, §2, de la directive 2019/1158.

64 ERA 1996, s 47.

65 ERA 1996, s 104C.

66 Art. 12 et 14 et préambule (41) de la directive 2019/1158.

67 Art. 9, § 1, de la directive 2019/1158 : «La durée de ces formules souples de travail peut faire l'objet d'une limitation raisonnable ».

68 Art. 9, § 3, de la directive 2019/1158.

69 O. Golynker, op. cit., 2015, p. 387.

70 Préambule (35) de la directive 2019/1158.

71 Discours de la Reine du 19 décembre 2019 : « Des mesures seront proposées pour encourager la flexibilité du travail, pour introduire le droit au congé pour les aidants non rémunérés »: https:// www.gov.uk/government/speeches/queens-speech-december-2019

72 The Queens' Speech - Briefing Background, notes p. 43 : https://assets.publishing.service.gov.uk / government/uploads/system/uploads/attachment data/file/853886/Queen s Speech December 2019 back ground briefing notes.pdf

73 "Employers should now always offer working from home as option, Matt Hancock says ", The Independent, 10 Juillet 2020. 
personnes bénéficiant d'une forme de travail flexible est passée de 23 à $27 \%$ entre 2005 et 2017). Ce chiffre est comparable à ceux d'autres pays européens ${ }^{74}$ (avec $25 \%$ de travailleurs à temps partiel au Royaume-Uni et $20 \%$ des salariés en télétravail, au moins partiellement). Cependant, les statistiques révèlent qu'il n'y a pas d'égalité des sexes face aux formules souples, puisque les femmes sont davantage susceptibles de travailler selon des modalités flexibles que les hommes ${ }^{75}$.

La jurisprudence démontre également un préjugé sexiste envers les salariés qui demandent des formules souples de travail. S'il a été établi très tôt que refuser d'accorder des modalités de travail flexibles pouvait constituer une discrimination indirecte fondée sur le sexe étant donné le nombre disproportionné de femmes demandant un changement de mode de travail pour raisons familiales ${ }^{76}$, les hommes peuvent également être directement victimes de discrimination fondée sur le sexe si une demande de modalités flexibles leur est refusée, alors que les demandes des femmes occupant le même poste ont reçu une réponse positive ${ }^{77}$. D'autres ont aussi critiqué le fait de s'appuyer sur les droits des travailleurs plutôt que sur ceux des familles, car cela met tous les salariés en concurrence les uns avec les autres.

Dans l'ensemble, il est clair que le Royaume-Uni est déjà largement conforme à la directive européenne. Un certain nombre de dispositions semblent même aller plus loin lorsque l'on considère le champ d'application de ce droit (tous les salariés en bénéficient) et la durée pendant laquelle les parents peuvent en bénéficier (jusqu'aux 17 ans de l'enfant). $\mathrm{Si}$ le Royaume-Uni devait aligner ses normes en matière de droit du travail, il pourrait envisager d'introduire des mesures qui supprimeraient la modification définitive du contrat de travail suite à une demande de flexibilisation du travail, en particulier un retour à des heures à temps plein ou à des horaires de travail plus conventionnels. Le droit introduit dans le point suivant de la directive européenne ajouterait cependant un nouvel élément à l'ensemble des droits favorisant l'équilibre entre vie professionnelle et vie privée.

\section{IV - LE CONGÉ D'AIDANT}

Comme le souligne la directive ${ }^{78}$ et comme c'est le cas au Royaume-Uni ${ }^{79}$, les aidants autres que les parents ont besoin que la loi leur accorde une plus grande attention, étant donné que les obligations familiales peuvent représenter un obstacle en termes d'avancement professionnel ou même de capacité à rester sur le marché du travail. Les chiffres démontrent que le nombre d'aidants augmente en raison du vieillissement de la population et de l'augmentation de l'espérance de vie ${ }^{80}$.

74 CIPD, Flexible Working in the UK, juin 2019, p. 3.

75 CIPD, MegaTrends - Flexible Working, janvier 2019, p. 15.

76 Hardy and Hansons Plc v Lax [2005] EWCA Civ 846.

77 Affaire non rapportée du Tribunal du travail. Voir BBC News, Father wins discrimination case : http:// news.bbc.co.uk/1/hi/scotland/1666173.stm

78 Préambule (27) de la directive 2019/1158.

79 G. James et E. Spruce, «Workers with Elderly Dependants: Employment Law's Response to the Latest Care-giving Conundrum », Legal Studies, 2015, vol. 35, p. 463.

80 Les derniers chiffres disponibles révèlent qu'entre 2001 et 2011, le nombre des aidants au Royaume-Uni a augmenté de 11\% : Carers UK, Facts about carers', Policy briefing : août 2019. 
Dans le monde du travail britannique, les aidants sont autorisés à demander des modalités de travail flexibles ou à prendre un congé sans solde en cas d'urgence ${ }^{81}$ et peuvent être protégés par la loi sur la discrimination si le traitement défavorable qu'ils ont subi s'explique par une discrimination fondée sur le handicap ${ }^{82}$. Cependant, la législation britannique n'est pour l'instant pas compatible avec la directive européenne, puisqu'il n'existe pas actuellement de congé d'aidant au Royaume-Uni.

Le droit européen exige que les aidants bénéficient de cinq jours de congé sans solde par an. A cette fin, un aidant est un travailleur qui apporte des soins personnels ou une aide personnelle à un membre de la famille ou à une personne qui vit dans le même foyer que le travailleur et qui nécessite des soins ou une aide considérables pour raison médicale grave ${ }^{83}$.

Sont ainsi définis comme "membres de la famille » : le fils, la fille, la mère, le père, le conjoint ou, lorsque la législation nationale reconnaît de tels partenariats civils, le partenaire civil d'un travailleur, même si la directive encourage les Etats membres à établir une liste plus exhaustive comprenant les frères et sœurs et les grands-parents ${ }^{84}$. La liste des membres de la famille pour lesquels il est possible de bénéficier d'un congé au Royaume-Uni devrait être définie si la loi britannique était modifiée. Mais dans l'ancien système autorisant le travail flexible, les parents et les personnes vivant à la même adresse étaient inclus. Les règles sur les congés d'urgence font référence aux personnes à charge.

Ce droit ne donne lieu à aucune rémunération, comme de nombreuses autres mesures favorisant l'équilibre entre vie professionnelle et vie privée (par exemple le congé d'urgence). Mais les rédacteurs de la directive européenne ont encouragé les Etats membres à mettre en place une telle rémunération ou allocation, afin de garantir le recours effectif à ce droit par les aidants ${ }^{85}$. Si ce droit devait être transposé au Royaume-Uni, il est peu probable que la rémunération soit obligatoire compte tenu des antécédents du gouvernement précédent en matière de congés sans solde (tel le congé d'urgence ou le congé parental).

L'application de ce droit est largement laissée à l'appréciation des Etats membres en ce qui concerne les conditions d'admissibilité (l'ancienneté par exemple), la période de référence pour exercer le droit (une année ou une autre période déterminée par la loi d'application) et la nécessité de documenter ou de prouver l'état de santé du parent nécessitant des soins ${ }^{86}$.

En conséquence, même si le Royaume-Uni mettait en œuvre la directive européenne, ce droit serait adapté pour tenir compte du contexte britannique. Que le contenu de la

81 ERA 1996, s 57A et 57B.

82 Affaire C-303/06, Coleman contre Attridge Law [1998] ECR I-00621, dans laquelle une secrétaire juridique avait été licenciée en raison de son absence du travail parce qu'elle s'occupait de son fils handicapé.

83 Art. 3, § 1, point d, de la directive 2019/1158.

84 Art. 3, § 1, point e, de la directive 2019/1158.

85 Préambule (32) de la directive 2019/1158.

86 Art. 6 de la directive 2019/1158. 


\section{LA DIRECTIVE 2019/1158 ET L'EFFET BREXIT}

directive ait influencé le gouvernement actuel ou non, le projet de loi sur le droit du travail prévoyait l'introduction d'un droit à un congé sans solde d'une semaine pour les aidants ${ }^{87}$.

\section{Conclusion}

S'il est possible que la directive 2019/1158 ne soit pas mise en œuvre au Royaume-Uni, la législation britannique sur l'équilibre entre la vie professionnelle et la vie privée a évolué dans le même sens et, à bien des égards, soit la réglementation britannique actuelle est similaire aux dispositions de la directive, soit elle accorde des droits encore plus généreux.

Dans le même temps, sur certains points, les parents qui travaillent gagneraient à ce que la législation s'aligne sur la directive 2019/1158, notamment en ce qui concerne le droit à une indemnité de paternité et le droit nouvellement créé à un congé d'aidant.

87 The Queens' Speech - Briefing Background, op. cit. : https://assets.publishing.service.gov.uk/government/ uploads/system/uploads/attachment data/file/853886/Queen's Speech December 2019 background briefing notes.pdf

\section{OXANA GOLYNKER}

Maître de conférences, Université de Leicester.

Thèmes de recherche: Equilibre vie professionnelle/vie privée, sécurité sociale et UE; Citoyenneté de l'UE.

\section{Publications:}

O. Golynker, " EU coordination of social security from the point of view of EU integration theory ", 2020, European Journal of Social Security, vol. 22(2), p. 110.

O. Golynker, "Family-Friendly Reform of Employment Law in the UK : an overstretched flexibility $», 2015$, Journal of Social Welfare and Family Law, vol. 37/3, p. 378.

\section{PASCALE LORBER}

Professeur, Université de Leicester.

Thèmes de recherche: Représentation collective et voix au travail, Loi sur l'égalité.

\section{Publications:}

P. Lorber et T. Jaspers, " Workers' Participation in Business Matters ", in T. Jaspers, F. Penning et S. Peters, European Labour Law, Intersentia, 2019.

P. Lorber, " Directive 2002/14/EC establishing a general framework for informing and consulting employees in the European Community ", in E. Ales, M. Bell, O. Deinert et S. Robin-Olivier (ed.), International and European Labour Law, Nomos, Hart publishing, 2018. 